\title{
Alterstice
}

Revue internationale de la recherche interculturelle

International Journal of Intercultural Research

Revista International de la Investigacion Intercultural

\section{LGBTQ racisés : frontières identitaires et barrières structurelles}

\section{Habib El-Hage et Edward Jin Lee}

Volume 6, numéro 2, 2016

URI : https://id.erudit.org/iderudit/1040629ar

DOI : https://doi.org/10.7202/1040629ar

Aller au sommaire du numéro

\section{Éditeur(s)}

Alterstice

ISSN

1923-919X (numérique)

Découvrir la revue

Citer cet article

El-Hage, H. \& Lee, E. J. (2016). LGBTQ racisés : frontières identitaires et barrières structurelles. Alterstice, 6(2), 13-27. https://doi.org/10.7202/1040629ar

\section{Résumé de l'article}

L'affirmation identitaire des personnes lesbiennes, gaies, bisexuelles, trans et queer issues de l'immigration (LGBTQ racisées) est en croissance constante au Québec. Malgré une reconnaissance publiquement affirmée de la diversité sexuelle, des discriminations de tout genre se trouvent banalisées sur le plan social et politique, au niveau national et international. Sur le plan politique, les démarches menées par des organisations ou des individus auprès des représentants politiques pour défendre les droits des personnes LGBTQ racisées sont peu nombreuses. Sur le plan social, la méconnaissance et l'incompréhension des besoins particuliers de ces personnes créent des situations d'injustice, de rejet, d'exclusion, d'homophobie, de transphobie et de racisme. Il existe peu d'information sur les ressemblances et les différences entre les barrières à l'intégration sociale (comme la difficulté d'accès au système de santé et services sociaux, confrontation au racisme et à la discrimination en emploi, etc.) vécues au Québec par les membres de la communauté LGBTQ en général et les personnes LGBTQ racisées. La documentation manque aussi concernant les recommandations que les personnes LGBTQ racisées jugent pertinentes pour lutter contre les discriminations multiples comme le racisme, l'homophobie et la transphobie, et pour améliorer ainsi leurs relations structurelles, interculturelles et intraculturelles. Cet article tente, à partir d'une étude exploratoire, d'apporter un éclairage sur les parcours divers, les identités multiples ainsi que sur les barrières vécues par les personnes LGBTQ racisées.
Ce document est protégé par la loi sur le droit d'auteur. L'utilisation des services d'Érudit (y compris la reproduction) est assujettie à sa politique d'utilisation que vous pouvez consulter en ligne.

https://apropos.erudit.org/fr/usagers/politique-dutilisation/ 


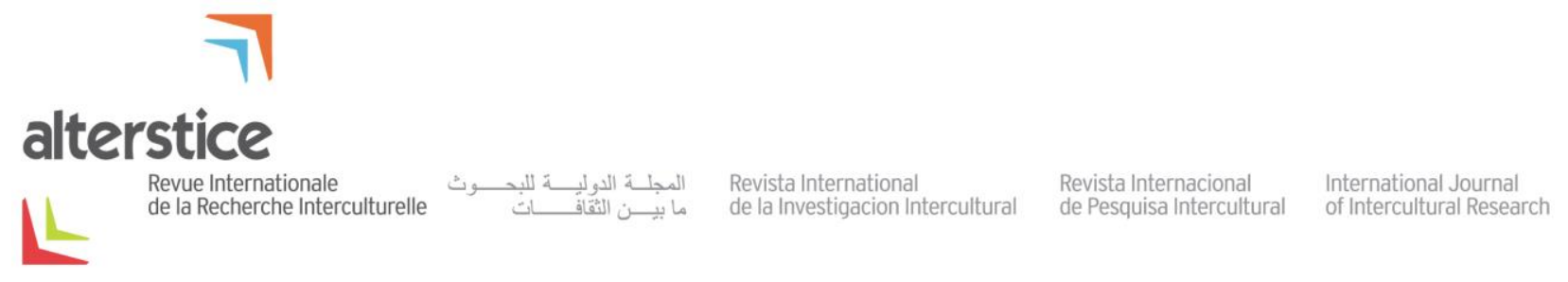

ARTICLE HORS THÈME

\section{LGBTQ racisés : frontières identitaires et barrières structurelles}

Habib El-Hage ${ }^{1,2,3}$ et Edward Jin Lee ${ }^{1,4,5}$

\section{Résumé}

L'affirmation identitaire des personnes lesbiennes, gaies, bisexuelles, trans et queer issues de l'immigration (LGBTQ racisées) est en croissance constante au Québec. Malgré une reconnaissance publiquement affirmée de la diversité sexuelle, des discriminations de tout genre se trouvent banalisées sur le plan social et politique, au niveau national et international. Sur le plan politique, les démarches menées par des organisations ou des individus auprès des représentants politiques pour défendre les droits des personnes LGBTQ racisées sont peu nombreuses. Sur le plan social, la méconnaissance et l'incompréhension des besoins particuliers de ces personnes créent des situations d'injustice, de rejet, d'exclusion, d'homophobie, de transphobie et de racisme. Il existe peu d'information sur les ressemblances et les différences entre les barrières à l'intégration sociale (comme la difficulté d'accès au système de santé et services sociaux, confrontation au racisme et à la discrimination en emploi, etc.) vécues au Québec par les membres de la communauté LGBTQ en général et les personnes LGBTQ racisées. La documentation manque aussi concernant les recommandations que les personnes LGBTQ racisées jugent pertinentes pour lutter contre les discriminations multiples comme le racisme, l'homophobie et la transphobie, et pour améliorer ainsi leurs relations structurelles, interculturelles et intraculturelles. Cet article tente, à partir d'une étude exploratoire, d'apporter un éclairage sur les parcours divers, les identités multiples ainsi que sur les barrières vécues par les personnes LGBTQ racisées.

\section{Rattachement des auteurs}

${ }^{1}$ Équipe METISS (Migration et ethnicité dans les interventions en santé et en services sociaux), SHERPA et CRIEC

2 Université de Sherbrooke, Sherbrooke, Canada

${ }^{3}$ Collège de Rosemont, Montréal, Canada

${ }^{4}$ AGIR [organisme d'aide venant en aide aux personnes LGBTQ immigrantes ou réfugiées], Montréal, Canada.

${ }^{5}$ École du travail social, Université de Montréal, Montréal, Canada.

\section{Correspondance}

elhageh@hotmail.com

\section{Mots clés}

LGBTQ; migrations; réfugiés; identité; intersectionnalité.

\section{Pour citer cet article}

El-Hage, H. et Lee, E. J. (2016). LGBTQ racisés : frontières identitaires et barrières structurelles. Alterstice, 6(2), 2540. 


\section{Introduction}

Cet article est le fruit d'une étude exploratoire réalisée auprès de personnes lesbiennes, gaies, bisexuelles, trans et queer (LGBTQ) racisées et d'intervenants du milieu de la santé et des milieux communautaires, dans le but d'explorer les perceptions et les expériences directes ou indirectes rencontrées par des personnes LGBTQ racisées vivant au Québec. Dans cet article, nous employons l'adjectif " racisé » pour désigner le processus de catégorisation et de désignation des individus non blancs ou, si l'on préfère, des " minorités visibles ". Nous utilisons le terme LGBTQ afin de signifier de multiples identités sexuelles et de genre tout en reconnaissant cette complexité. Dans ce contexte, les termes " queer » et " trans » sont chargés de sens théoriques et politiques (voir dans ce sens les écrits de Cohen, 1998, et Haritaworn, 2012). Le terme " queer » est un terme choisi pour désigner les personnes non-hétérosexuelles. Le terme " trans » est un terme parapluie pour désigner les personnes qui s'identifient à leur genre différemment du sexe assigné à la naissance. Nous avons voulu dresser les grandes lignes du phénomène étudié et apporter des réponses aux questions suivantes : quels sont les empêchements ou les barrières qui entachent le quotidien des personnes LGBTQ racisées? De quelle manière ? Quelles en sont les conséquences sur les relations interculturelles ? Comment la société d'accueil doit-elle répondre aux différentes barrières qui empêchent la prise en considération des réalités des personnes LGBTQ racisées ? De quelle manière ces barrières influencent-elles la participation citoyenne? Nous avons voulu dégager les facteurs structurels, communautaires et personnels qui aident ou, au contraire, entravent une réelle inclusion des personnes LGBTQ racisées au sein de la société québécoise.

En explorant l'expérience des personnes LGBTQ racisées et celle d'intervenants à partir de leur point de vue, nous pourrons mieux saisir leur réalité et leurs besoins. Nous pensons que les résultats obtenus éclaireront les personnes intervenantes et professionnelles et sauront guider l'élaboration de services plus adaptés aux besoins, notamment en ce qui a trait à la lutte contre les discriminations multiples dans le contexte particulier des personnes LGBTQ racisées.

\section{Contexte historique}

Il est actuellement connu que les discriminations basées sur la diversité sexuelle sont souvent perçues comme étant qualitativement différentes d'une société à l'autre. Certes, il y a des niveaux inégaux d'acceptation sociale et de violence contre les personnes LGBTQ à travers le monde. Cependant, les diverses formes de violence homophobe et transphobe qui se manifestent à l'échelle mondiale ont les mêmes racines historiques (Lee, 2015). En ce sens, la gestion coloniale des populations a marqué, au $19^{\mathrm{e}}$ siècle et après, les vies intimes des sujets tout en criminalisant les rapports sexuels non normatifs (Alexander, 2005 ; Gupta, 2008 ; Lee, 2015).

Aux $19^{\mathrm{e}}$ et $20^{\mathrm{e}}$ siècles, les grands empires européens avaient confirmé leur domination sur l'ensemble des continents africain, asiatique et sur les Amériques (Lowe, 2006). Au temps où les dirigeants coloniaux gouvernaient, ils organisaient les rapports entre les sujets en instaurant des systèmes de normes conformes à l'idéologie des grandes puissances en présence (Lowe, 2006). Les rapports sexuels étaient organisés et codifiés par les institutions politiques, religieuses et éducatives (Stoler, 2006). Les dirigeants coloniaux ont défini la sexualité et le genre non normatifs comme étant une déviation à bannir, justifiant ainsi la surveillance et la criminalisation des personnes ayant une telle sexualité (Gupta, 2008; Lee, 2015). Cet état de faits a continué après l'affranchissement des anciennes colonies (Alexander, 2005). Certains pays asiatiques et africains ont renforcé après leur indépedance l'ordre social hétéronormatif ${ }^{1}$, cisnormatif $^{2}$ et patriarcal par un discours sur les "valeurs familiales » et sur la promotion du mariage monogame hétérosexuel, tout en criminalisant la sexualité non normative (Alexander, 2005 ; Alexander et Mohanty, 1997; Ekine, 2013 ; Lee, 2015). Les lois coloniales sont restées, puis intégrées comme faisant partie des normes culturelles du pays. Ce contexte historique et économique sert de "base structurelle "à la violence quotidienne contre les personnes LGBTQ (Ossome, 2013).

\footnotetext{
${ }^{1}$ L’hétéronormativité s'inscrit dans la société par la reproduction institutionnelle et discursive de l'hétérosexualité comme « la norme, la bonne sexualité, naturelle, morale, pure et propre... bénéfice de privilèges comme la famille, le mariage, sa représentation dans les manuels scolaires, au grand écran (etc.) » (Ricard, 2011, p. 81).

${ }^{2}$ Selon Enriquez (2013), la cisnormativité « modèlerait les politiques et les pratiques des individus et des institutions, de manière à rejeter l'existence ou la visibilité des personnes trans » (p. 183).
} 
Pour une meilleure compréhension du contexte historique de la diversité sexuelle et de genre au Québec, un point de départ important consiste à reconnaître l'organisation sociale qu'avaient de nombreuses Premières Nations avant le contact colonial. À cette époque, de nombreuses communautés autochtones vivaient harmonieusement avec une diversité sexuelle et de genre au sein de leurs communautés. Chaque communauté utilisait une terminologie spécifique désignant des personnes homosexuelles comme étant des médiatrices, des enseignantes ou des guides spirituelles (Meyer-Cook et Labelle, 2004). Les sociétés autochtones valorisaient l'esprit communautaire et affichaient des lois et règles flexibles de mariage, notamment entre personnes du même sexe (Carter, 2008).

Avec la venue des puissances européennes, les valeurs occidentales portant sur la sexualité non normative ont déformé les expressions indigènes de la sexualité et du genre. Les colons français et britanniques ont imposé leurs valeurs spirituelles et organisationnelles aux communautés autochtones. En 1892, le gouvernement canadien criminalisait les activités sexuelles entre personnes du même sexe (Roy, 2013). Pour la première fois, la loi sur l'immigration canadienne de 1906 a inclus comme catégorie d'exclusion les personnes ayant commis un crime de " turpitude morale ", ce qui incluait l'expression du genre et la conduite sexuelle dite " immorale " (Lee, 2015). En 1927, la pathologisation et la criminalisation de l'homosexualité sont apparues dans la loi sur l'immigration canadienne, loi qui mentionnait explicitement l'exclusion des personnes identifiées comme des déviants sexuels (White, 2010).

L'émergence de l'homosexualité comme identité déviante est la preuve de la manière dont l'Église, le milieu médical et l'État canadien ont promu le contrôle social et ont insufflé chez la population une méfiance envers les personnes jugées comme étant des dégénérées et des déviantes sexuelles (Kinsman, 1998). Demczuk et Remiggi (1998) stipulent que "l'occultation sociale a été en effet l'une des principales formes de répression de I'homosexualité et du lesbianisme pendant la majeure partie du $20^{\mathrm{e}}$ siècle, et ce, non seulement au Québec, mais presque partout dans le monde » (p. 13).

Au Québec, à partir des années 1960, un tournant historique - connu sous le nom de Révolution tranquille - a permis une transformation des institutions religieuses en institutions gérées par l'État. Un mouvement de libération sexuelle a pu aussi être observé et aida grandement à l'acceptation des sexualités non normatives (Roy, 2013). Un discours affirmé émergea avec le bill omnibus de 1967, qui a eu pour effet la décriminalisation de I'homosexualité dans les espaces privés. En 1977, le Québec incluait la protection de l'orientation sexuelle dans la Charte québécoise des droits de la personne. En 2002, le Québec a instauré un régime d'union civile pour les couples du même sexe. En ce sens, le progrès sur les plans juridique et politique continue. Certains groupes de défense des droits des minorités sexuelles et de genre militent en faveur d'une réelle égalité sociale des personnes LGBTQ, spécifiquement celles issues de l'immigration ou réfugiées, dans le but notamment d'éliminer la violence à leur égard et de les aider à sortir de la marginalité.

\section{Cadre théorique}

Nous présentons maintenant les théories, approches et concepts que nous allions dans notre cadre théorique, notamment l'intersectionnalité, le processus de racisation et l'identité culturelle.

\section{Intersectionnalité}

Il existe plusieurs modèles d'autoreprésentation des "identités ethnosexuelles ", ce qui empêche de consacrer une identité particulière comme étant celle de toutes les personnes LGBTQ racisées (Chbat, 2011). II est donc nécessaire de saisir ces identités dans leur multiplicité, surtout eu égard aux expériences communes liées à la migration, au genre et à d'autres facteurs, ce que nous tenterons de faire dans les prochaines sections. Ces identités plurielles constituent l'une des raisons pour lesquelle nous avons mis en lumière le concept d'intersectionnalité.

L'analyse intersectionnelle permet de mieux comprendre l'identité en prenant en considération ses diverses facettes (Crenshaw, 1996). Cette théorie transdisciplinaire considère la complexité des identités et des inégalités sociales par une approche intégrée, en refusant le cloisonnement et la hiérarchisation des grands axes de la 
différenciation sociale et en postulant que l'interaction des multiples systèmes d'oppression peuvent reproduire des inégalités sociales (Bilge, 2009 ; Lee et Brotman, 2011). En considérant diverses catégories telles que le genre, la " race » ou la classe comme des structures se croisant plutôt que comme des catégories d'oppressions distinctes, ce concept permet non seulement d'analyser les expériences vécues par les individus selon leur positionnement sur divers axes de domination ou d'oppression et leur combinaison, mais explicite également les contradictions qui existent dans les situations de privilèges (Bilge et Denis, 2010).

La plupart des études ont conclu que les personnes LGBTQ racisées appartiennent de manière conflictuelle à trois communautés, soit la société d'accueil, les communautés ethnoculturelles et les communautés LGBTQ, dont les normes tout comme les valeurs ou croyances liées à la sexualité ou aux origines ethniques divergent (Espin, 1997). Ainsi, certaines vont se définir en faisant référence parfois à leur sexualité, parfois à leurs origines, parfois aux deux (Kapac, 1998).

\section{Les processus de racisation et l'identité culturelle}

Il existe un processus de racisation lorsque certaines caractéristiques sont retenues afin de décrire et de classifier d'autres humains, surtout lorsque des différences liées au corps ou encore à la culture sont jugées par la majorité comme étant autres, particulières et inférieures (Chbat, 2011 ; Guillaumin, 1972 ; Miles, 2003). Les personnes LGBTQ immigrantes et ou racisées seront autrement marginalisées relativement à leur racisation (Espin, 1997). Bien que la société québécoise et canadienne valorise dorénavant l'égalité homme-femme ou l'ouverture aux personnes LGBTQ, celles-ci ne se concrétisent pas toujours dans la réalité (Gagné et Chamberland, 2008). Sur ce point, il semble qu'une certaine "stratification sociale " soit présente, délimitant une frontière entre les " désirables » et les " indésirables ». En effet, seules certaines personnes LGBTQ seraient jugées désirables par la société, surtout les hommes gais blancs de classe moyenne : les autres, surtout les personnes trans, immigrantes et racisées, sont exclues (Duggan, 2003 ; Puar, 2007 ; Roy, 2013).

Ainsi, certaines barrières structurelles existent, notamment le racisme systémique, et ce, malgré le fait que l'Occident se dise et se veuille plus « évolué » que ces « ailleurs » qui oppriment les personnes LGBTQ racisées ou les femmes (Bilge et Denis, 2010). Par ailleurs, il y a des liens entre le racisme et la pauvreté ainsi que les barrières systémiques concernant l'usage des services sociaux, du système de santé et de l'éducation (O'Brien et al., 2005). Les processus de racisation façonnent donc le développement de l'identité des personnes LGBTQ racisées.

Nous avons vu que les identités de personnes LGBTQ racisées sont multiples et complexes, et ne peuvent être généralisées (Chbat, 2011). Les parcours des LGBTQ racisés sont variés, mais les préjugés, la fétichisation ainsi que I'invisibilité existent dans les communautés LGBTQ, ce qui peut créer un sentiment d' « objectification » et d'exclusion (Roy, 2013).

\section{Méthodologie}

Nous avons opté, comme processus méthodologique, pour une démarche qualitative, afin d'effectuer une exploration en profondeur d'un sujet complexe. Nous avons utilisé une méthode d'entretien en groupe de discussion (focus group). Nous avons rencontré trois groupes, dont la taille variait entre 3 et 8 personnes. L'échantillon était composé de 23 personnes. Deux groupes étaient constitués d'informateurs avec de riches expériences d'intervention auprès des personnes immigrantes LGBTQ. Le troisième groupe était composé de personnes LGBTQ issues de l'immigration ou réfugiées, trois critères principaux ayant orienté la sélection des candidats de ce groupe :

- I’origine ethnique,

- le statut d’immigrant de première ou de deuxième génération ou de réfugié,

- I'orientation sexuelle et l'identité du genre.

Le choix de mener des entretiens de groupe était motivé par la volonté de faciliter l'interaction des participants, qui pouvaient construire sur le discours des uns et des autres. L'hétérogénéité concernant les lieux d'appartenance et 
les lieux des pratiques des intervenants visait la mise en rapport de perspectives et d'expériences différentes ainsi que l'examen des convergences et des divergences de position quant aux cadres d'analyse du phénomène et quant aux solutions à privilégier. Les principaux thèmes abordés dans les entretiens auprès des personnes LGBTQ racisées étaient :

- les expériences prémigratoires et l'expérience du projet migratoire,

- les expériences dans la société d'accueil.

Les principaux thèmes abordés dans les entretiens auprès des responsables d'organismes communautaires et des intervenants psychosociaux étaient :

- les services offerts à la population LGBTQ racisée,

- la perception générale de la part de la société d’accueil à l'égard des personnes immigrantes LGBTQ racisées, les stéréotypes et préjugés,

- les facteurs de protection et de fragilisation des personnes LGBTQ racisées.

Les entretiens avec les répondants LGBTQ racisés visaient à leur donner une liberté de parole quant à la représentation de leur vécu. Les entretiens avec les intervenants et les responsables des différents organismes visaient à cerner les difficultés éprouvées par les personnes LGBTQ racisées et les moyens ou solutions systémiques à apporter dans le cadre du service fourni.

Étant donné le caractère exploratoire de l'étude, l'ensemble du recrutement des participants et participantes a été effectué par l'organisme AGIR. Dans le but de protéger l'identité et le statut de plusieurs personnes qui ont participé, nous avons accepté, à la demande de certaines d'entre elles, de ne pas exiger de données personnelles liées à certaines caractéristiques comme le sexe, l'âge ou le statut d'immigration. Nous avons préservé leur anonymat en leur attribuant des prénoms fictifs dans les citations.

\section{Synthèse des données}

Cette section présente les perspectives des personnes rencontrées dans le cadre d'un groupe de discussion mettant en lumière l'expérience de vie dans la société d’origine, l'expérience du trajet migratoire et l'expérience dans la société d'accueil.

\section{Société d'origine et rapport à la diversité sexuelle}

Le parcours personnel des répondants et répondantes rencontrés, qui se caractérise par des références à un vécu plein d'embûches, varie d'une personne à l'autre. Les variations peuvent être regroupées en fonction de trois aspects importants : le caractère douloureux du parcours, la préservation de l'honneur familial ${ }^{3}$ et la lutte contre I'homophobie et la transphobie.

\section{Un parcours empreint de défis}

Interrogés sur les manifestations de nature homophobe et transphobe, plusieurs participants affirment avoir vécu des expériences difficiles dans leur pays d'origine. Puisque la plupart viennent de sociétés hétéronormatives et cisnormatives, les personnes rencontrées ont dû affronter une trajectoire pleine d'embûches. Elles témoignent d'un vécu dans une société machiste empreinte de préjugés, de stéréotypes et de violence sous formes diverses,

\footnotetext{
${ }^{3}$ La notion de l'honneur a été traitée par plusieurs auteurs de différentes disciplines. L'honneur constitue un élément central dans toute organisation sociale. Selon Pitt-Rivers (1966) «toute société, ancienne ou moderne, est fondée sur un code de l'honneur et de la honte, qui influence les relations sociales au sein de la famille et de la communauté.» (p. 22). Selon lui, "l’honneur reflète les valeurs morales d'une société, que tout pouvoir politique prétend incarner. Ce dernier se donne le droit de dicter le bien et de punir le mal, d'attribuer l'honneur ou d'infliger le déshonneur aux individus, selon qu'ils se conforment ou non aux comportements souhaités » (p. 22).
} 
que ce soit au sein du voisinage, à l'école, dans la famille ou ailleurs. L'histoire de Liz, qui a souffert d'intimidation dans son pays d'origine, caractérisé par le « machisme », est frappante :

My country of origin is one of "machisma"; they can't stand somebody who is a little feminine. Ever since I can remember, I suffered from bullying. When I was in elementary school, I was beat up by different kids for being a little LGBT. (Liz)

Même la police n'offrait pas la protection nécessaire, se plaindre n'étant pas pris au sérieux, comme en témoigne Maria à propos de ses voisins agressifs :

The neighbors used to be aggressive toward me. Even the police don't do nothing. When you complain, they don't take it seriously. (Maria)

Les personnes rencontrées ont exprimé une douleur de vivre l'orientation sexuelle ou l'identité de genre dans leur société d'origine. Même dans la famille proche, le rejet et la violence étaient présents :

Nous, les jeunes qui nous sentions différents de la société d'origine, nous nous détachions un peu. Les parents percevaient certains comportements comme problématiques, du fait qu'il y a des comportements qui sont considérés comme conflictuels entre les deux cultures. Donc, il y a vraiment eu des tensions, surtout lorsque je me suis affirmée comme homosexuelle. C'est vu comme impossible d'être gai dans la famille, la communauté. (Imane)

\section{L’honneur familial}

Exprimer son orientation sexuelle ou son identité de genre est une tare dans un contexte où il faut préserver I’honneur familial. Plusieurs répondants ont révélé l'importance pour leur famille de cette notion d'honneur. Cette notion prenait plusieurs formes, le lexique différant d'une société à l'autre. Pietro évoque la honte vécue et exprimée dans sa société et par sa famille. Son témoignage rejoint celui de plusieurs personnes rencontrées :

La communauté est très serrée. Es una verguenza para la familia, una cuestion de honor. [C'est une honte pour la famille. Une question d'honneur.] (Pietro)

\section{La lutte contre l'homophobie et la transphobie}

La lutte contre I'homophobie et la transphobie est longue, complexe, difficile et dangereuse. Certains enjeux politiques entrent en considération dans le jeu des droits et libertés. Certains répondants ont expliqué les façons dont ils et elles ont lutté contre l'homophobie et la transphobie dans leur pays d'origine. Ces répondants ont également évoqué l’histoire complexe liée à la dynamique de la diversité sexuelle et de genre même lorsque, dans de rares cas, le soutien de la famille est acquis. Julia, par exemple, affirme que l'hostilité venait surtout de la société.

My mom and my sister still support me. They supported me in the sense that, since I "came out", my mom has always respected my decision, she understands me. She always give me advice and my sister also still defend me. It feels good. Because, not many people do have members of their family supporting them. Even if my family supports me, the broader society was still hostile. (Julia)

La complexité exprimée par Pietro relève davantage du harcèlement vécu, de l'homophobie et de la violence exercée par des membres de la police contre lui et ses amis. Selon lui, le fait d'être une personne LGBTQ racisée suffisait (selon la police) pour avoir ce traitement. Même après avoir filmé avec ses amis cette violence, les choses n'ont pas changé beaucoup :

For me the tacit, supportive groups were my friends. We started out as 5 and ended up being 20. We would find ways to better defend ourselves from other people. We went several times to the city townhall and complained about the police. The police was harrassing us, insulting us, some even wanted us to have sex with them. The city president ignored us and even one of us got beaten afterward. They said we deserved it because we are like we are: LGBTQ racisés, faggot. Me myself, I even sued some of the police officers, we filmed them and recorded their actions. They didn't like it. We went to the human rights commission. Things have changed a bit, but not so much. (Pietro) 


\section{La perception de la société québécoise}

La perception de la société hôte par les futurs arrivants est marquée par une idéalisation de la reconnaissance et de l'ouverture à l'égard de l'Autre. Choisir de quitter la société d'origine paraît une solution à privilégier pour plusieurs personnes. Mais plusieurs obstacles s'imposent. Lors de nos entretiens de groupe, nous avons constaté que la réalité vécue dont témoignaient les personnes répondantes ne correspondait guère à la réalité qu'elles avaient imaginée. Tous avaient vécu un trajet migratoire particulier, les situations étant différentes d'une personne à l'autre, le départ pouvant s'être fait en famille ou en solo, par exemple. II n'est pas rare que le trajet vers Montréal ait été discontinu et entrecoupé par de multiples arrêts. À l'arrivée au Québec, le rêve et la réalité entrent en collision. Le quotidien empreint de discrimination et d'embûches a des conséquences certaines sur la vie des personnes en situation de vulnérabilité. Malgré cette dure réalité, les répondants nous parlent de plusieurs solutions pleines de sens.

\section{Le trajet migratoire}

Les témoignages de rejet ne sont pas l'exception : avoir une orientation sexuelle ou une identité de genre hors norme n'était souvent pas bien accueilli par les membres de la famille. Pour la plupart, partir était la seule option qui s'offrait. Pour d'autres, des motifs différents étaient à l'origine du projet migratoire. L'image de la société canadienne ou québécoise est très flatteuse à l'étranger, particulièrement en ce qui a trait au respect des droits des personnes LGBTQ. Rosa et Varna exposent leurs perceptions respectives :

En dehors du pays, le Canada paraît comme un pays juste, sans préjugés, parfait, tolérant, où la phobie n'existe pas. (Rosa)

En dehors du Canada, le pays est vu comme un paradis. C'est complètement différent quand tu es au pays. (Varna)

Si ces témoignages révèlent la réputation enviable du Canada comme société d'accueil à l'égard des personnes LGBTQ, le choix de venir au Canada et au Québec est parfois lié au parcours de vie des parents. Dans le cas d'Imane, c'est le contexte politique dans le pays d'origine qui a poussé ses parents à partir. Voici comment elle explique la décision de ses parents :

Mon père est venu d'un petit village du Moyen-Orient, et il voulait devenir professeur d'université. II a été parrainé pour venir ici après la guerre civile, il se trouvait devant une impasse. Donc, ils sont [mes deux parents] venus pour des raisons d'oppression politique. (Imane)

\section{Du beau rêve à la dure réalité}

La majorité des répondants et répondantes affirment que, si le désir de venir au Québec relevait davantage du rêve et de l'idéalisation, la société réelle n'a pas été à la hauteur de leurs espoirs. Que ce soit pour un travail, pour un logement, un hébergement ou même pour une visite chez le médecin, les barrières sont multiples. Varna idéalisait la société canadienne à travers le prisme de certaines images véhiculées dans son pays d’origine. Voici ce qu'elle raconte :

Quand tu n'as pas de statut, tu te rends compte comme c'est dur. Surtout au Québec, où tu ne peux pas exercer ta profession, du fait que tes acquis ne sont pas reconnus... Pour survivre, tu dois trouver une job de crap. Tu « struggle " davantage parce que les jobs que tu peux occuper sont mal payées, et les conditions sont précaires. (Varna)

Le désenchantement s'installe rapidement. Les témoignages de Varna et d'Hassan sont très révélateurs des événements marquants qu'ils ont vécus lors de leur établissement, et surtout lors du contact avec la société. Hassan raconte les difficultés qu'il a rencontrées au travail :

Le système fait que les demandeurs d'asile peuvent facilement intégrer le processus et difficilement en sortir. C'est comme la mafia, et ça s'ajoute aux stigmatisations des collègues de travail, qui discriminent déjà les citoyens et résidents permanents. (Hassan) 


\section{La discrimination systémique des personnes racisées et immigrantes}

Lors des entrevues, les répondants et répondantes ont amplement commenté la réalité du quotidien empreint d'embûches. Ils ont fait état, entre autres, de considérations systémiques et de difficultés concernant le statut d'immigration et la reconnaissance de leurs qualifications. Certaines personnes ont pu relever des politiques et des pratiques qui perpétuent la discrimination systémique. Pietro exprime son désarroi devant la subtilité de la discrimination rencontrée lors de la recherche d'un logement :

Je crois que la discrimination, c'est très subtil. On a le numéro d'assurance sociale qui montre qu'on est réfugié [...]. Tout le monde sait que le numéro 9, c'est pour " réfugié ", c'est pour " temporaire " [...]. Alors, bien sûr que si tu demandes un logement, il [le locateur] te voit et il sait que tu es un réfugié. [...] Si ton numéro d'assurance sociale commence par 9 , il te demande une garantie. (Pietro)

Bien que les sujets décrivent le Québec comme une société des droits et libertés fondamentales de la personne, plusieurs relèvent une discrimination en milieu de travail. Ce sont, selon eux, les personnes racisées qui sont le plus touchées, du fait des marques apparentes de leur différence religieuse ou phénotypique.

J'ai appliqué [pour un travail] à un centre d'appels grâce à une amie, et c'est dans ce travail que j'ai réalisé la dynamique du pouvoir des autorités d'immigration... Dans la boîte, 80 \% des gens à temps partiel sont des immigrants ou des fils et filles d'immigrants. Par contre, pratiquement tous les administrateurs et les employés à temps plein sont des Blancs. Et quand il s'agit de faire des appels, les gens avec des accents sont davantage harcelés. (Asif)

\section{La perception de l'homophobie et la transphobie au travail}

Bien que certains répondants décrivent la société d'accueil comme une société ouverte à la diversité sexuelle et de genre, plusieurs d'entre eux relèvent une discrimination à l'embauche et au travail. Selon eux, la diversité sexuelle et de genre n'est pas bien accueillie dans certains milieux de travail. Plusieurs ont partagé des expériences d'homophobie et de transphobie pendant la recherche d'emploi et le processus d'embauche.

Pendant le processus d'embauche, bien sûr qu'il [l'employeur] ne peut pas te demander si tu es gai. Quand tu arrives là-bas et que tu es ouvertement gai, tu dis : " Je suis homosexuel. " J'ai eu des problèmes, oui, parce qu'une fois j'ai touché un gars sur l'épaule et il a dit : « Ne me touche pas! »(Carlos)

\section{L'exclusion et ses répercussions}

Plusieurs répondants et répondantes ont partagé leur expérience de l'homophobie et de la transphobie au sein de leur communauté immigrante ou racisée. Par exemple, Imane a expliqué les conséquences de la pression exercée par les membres de sa famille.

Quand j'ai nommé mon homosexualité [à mes parents], je ne pouvais plus rester. (Imane)

Je ne fréquente pas les gens de ma communauté culturelle, parce qu'ils ont les mêmes valeurs et comportements qu'au Mexique. (Pietro)

L'exclusion pour des raisons d'orientation sexuelle entraîne parfois un questionnement et une réappropriation identitaires. En ce sens, une stratégie de correction de stigmate est opérée. Pour certains et certaines, cela signifie quitter la maison pour de bon. Pour d'autres, couper les liens avec la communauté d'origine devient nécessaire. L'intervenante Claire raconte l'isolement vécu par les personnes LGBTQ racisées :

Déjà, dans le groupe d'immigrants qui arrivent, les LGBTQ sont isolés. Arriver comme demandeur d'asile LGBTQ, ça ne veut pas dire que tu vas être confortable de t'identifier comme gai ou lesbienne et de partager ton vécu. $Y$ a pas non plus une généralité là-dessus, donc le demandeur d'asile s'isole. (Claire)

La continuité des gestes homophobes ou transphobes au sein des communautés racisées rend difficile la solidification des liens avec la famille biologique. Selon l'analyse d'un répondant, la persistance des valeurs de la 
société d'origine impose une continuité des mécanismes de survie. Les stratégies d'évitement continuent même lorsque la famille biologique est absente. En ce sens, les intervenants des organismes d'aide participent aux stratégies d'évitement afin de bien desservir les personnes LGBTQ racisées :

Dans un cas où on intervenait avec la communauté camerounaise, même si les membres de la famille de cette personne-là [personnes LGBTQ camerounaise] n'habitaient pas à Montréal [...], l'accompagnement devait être fait sous couvert pour éviter que notre présence ne perturbe l'environnement social de la personne dans la communauté. (Annie)

En plus, certaines exclusions présentes dans le milieu LGBTQ relèvent de la réalité des personnes racisées. Imane exprime son désarroi devant le rejet exercé à son égard du fait qu'elle soit racisée :

Mais lorsqu'une lesbienne recherche un modèle de lesbienne du côté blanc québécois, tu ressens un rejet parce que ton nom est différent, tes cheveux font que les gens te rejettent comme " autre "... Je suis racisée parce que je mange différemment, je mange de l'ail. Aussi, je suis victime de commentaires super racistes. (Imane)

Certains intervenantes et intervenants communautaires constatent la présence d'exclusion et de racisme au sein des communautés LGBTQ. Ils explorent les conditions qui perpétuent l'exclusion des personnes LGBTQ racisées au sein des multiples organismes. Jean-Michel explique le rapport de pouvoir entre les différentes communautés LGBTQ :

Je remarque qu'il y a peu de diversité culturelle et ethnique au sein des organismes desservant les personnes LGBTQ, à moins que l'organisme desserve spécifiquement des populations racisées. Dans les organismes LGBTQ de la région de Montréal, il y a très peu d'intégration des personnes racisées dans la prise de décision, la participation et l'emploi dans l'organisation. On peut d'ailleurs remarquer une inégalité dans la répartition des ressources... En général, dans le milieu LGBTQ québécois, il y a une résistance et très peu de questionnements faits sur le rapport de pouvoir qui exclut les personnes non blanches. (Jean-Michel)

Cependant, pour Philippe, il existe une affinité et des raisons historiques de financement entre les organismes LGBTQ, ces organismes fonctionnant en réseau pour des raisons économiques et de survie :

Il y a très peu de financement pour les organismes communautaires LGBTQ... Je pense que la réalité actuelle dans le milieu LGBTQ est le résultat d'un rassemblement qui s'est fait par affinités, intérêts et complicité, pas nécessairement par racisme. (Philippe)

Même pour avoir accès aux services sociaux et aux soins de santé, les problèmes sont grands. Claire, intervenante dans ce milieu, évoque la désorganisation et l'insensibilité des autorités à la question de l'homophobie et de la transphobie en contexte multiethnique. Certaines structures d'aide peuvent créer des effets pervers. Par exemple, Suzanna raconte son expérience d'examen médical durant lequel elle s'est sentie jugée par rapport à son identité sexuelle. Claire décrit la cohabitation difficile dans des centres d'hébergement pour réfugiés, où on trouve des personnes LGBTQ et des hétérosexuels sous le même toit :

Les personnes peuvent être hébergées, mais elles le seront avec tout le monde et donc avec des personnes qui peuvent avoir des comportements très homophobes... On n'a pas un service spécialisé pour offrir un autre type d'hébergement... C'est difficile pour la personne, car elle arrive dans un pays où elle croit que cette situation sera finie, et finalement elle se perpétue en arrivant, les premiers mois. (Claire)

Georges, intervenant dans un cégep, raconte l'isolement vécu par les jeunes LGBTQ racisés dont, à cause de raisons complexes, l'orientation sexuelle reste tacite. Cette stratégie vise à réduire le stress de la confrontation avec la famille et la communauté. Georges nous raconte l'histoire d'un étudiant du Moyen-Orient qui avait eu des problèmes pédagogiques. La pression exercée sur son orientation sexuelle était grande. Sa sexualité est devenue un facteur de fragilisation dans sa vie et dans sa capacité de faire face aux éléments stressants.

One student from Middle East was having some problems concentrating dealing with course load and coursework... He was the older son in his family and his family was looking to him to be successful to help support the family... I don't think he was completely out, but they were aware there was something... and there was a pressure to go in a certain direction [to be 
heterosexual]... The weight of the family and the future of the family [was] on his shoulders... His sexuality then became a factor that affected his life and his ability to handle certain stressors. (Georges)

Hassan analyse clairement sa situation en tant que demandeur de statut de réfugié. Originaire d'Asie du Sud-Est, il a quitté son pays d'origine à cause d'une violence homophobe qu'il a subie.

Ma vie est en pause à cause de mon attente de statut. C'est une bureaucratie déshumanisante, on te fait sentir comme un rien. Ils t'imposent des limites... (Hassan)

\section{Les contributions des personnes LGBTQ racisées}

Les répondants et répondantes trouvent difficile de vivre en fonction de plusieurs barrières et discriminations. Mais ils considèrent malgré tout qu'il est important de contribuer à la société. Miriam considère que le dialogue ouvert entre les personnes LGBTQ racisées et la communauté d'origine permettra aux membres de cette dernière de mieux s'intégrer à la société d'accueil :

Le fait de pouvoir partager sa réalité avec la société québécoise, cela permet d'ouvrir les discussions sur ces causes. Ça aide à enrichir une société qui se veut inquiète des droits humains... II faut qu'il y ait un dialogue, et c'est aussi une responsabilité que les immigrantes ou réfugiées ont pour pouvoir aider à intégrer la société. Il y a une contribution culturelle et humaine à faire. (Miriam)

Pour Madeleine, la contribution des personnes LGBTQ racisées serait utile sur le plan politique, car leurs points de vue diversifiés enrichissent les discussions, surtout sur le plan politique :

They can bring perspectives that are not necessarily heard. I think that they are more politically-minded than we think... A lot more refugees and immigrants tend to be a lot more outspoken than we take for granted and so they add on the discourse and disrupt the white discourse. (Madeleine)

\section{Les solutions proposées par des personnes LGBTQ racisées}

Pour les personnes rencontrées, plusieurs solutions réalistes peuvent rendre le quotidien plus facile. Elles nomment la pertinence de miser sur l'influence des médias, de continuer l'éducation auprès des jeunes, de rendre accessibles des services spécialisés ou même de mettre en place un lieu regroupant différents services.

\section{Améliorer l'image dans les médias}

Parmi les solutions proposées, notons la déconstruction de l'image défavorable présentée dans les médias et répandue parmi la population. Cette déconstruction pourrait notamment s'appuyer sur des campagnes de sensibilisation à la diversité sexuelle et du genre et à l'importance du vivre ensemble qui comporteraient des modèles immigrantes ou racisées. Pour Sophie, une responsabilité incombe aux personnes LGBTQ racisées dans la modification de cette image déformée de leur situation. Pour arriver à changer cette image, Lucas propose pour sa part de recourir à des moyens efficaces tels que des formations, des outils de communication (vidéo, feuillets).

\section{Accroître la formation des intervenants de proximité}

Un participant souligne l'importance d'une formation sur la complexité de l'identité LGBTQ immigrante et sur les barrières à promouvoir leur bien-être psychosocial. Cette formation doit viser des personnes qui offrent des services publics, des représentants des organismes venant en aide aux personnes immigrantes et racisées.

Il y a un manque de coordination des services, pour être dirigé vers les bons services. Souvent, les organismes n'arrivent pas à rejoindre les gens qui ont vraiment besoin de leurs services, pour le processus d'immigration, mais aussi pour leur bienêtre psychosocial. (Pietro) 
Dans le même registre, l'information et l'éducation des communautés immigrantes et racisées font partie des solutions à envisager. Une intervenante souligne l'importance de la communication entre entités diverses, du dialogue et de la sensibilisation :

Je pense qu'il est important de continuer le dialogue entre les personnes LGBTQ racisées et les organismes et institutions de divers domaines (santé, éducation) afin de refléter le pouls qu'on prend de la situation. (Madeleine)

Quelques représentants des organismes communautaires au sesrvice des personnes LGBTQ racisées et immigrantes suggèrent une approche structurelle et fondée sur l'organisation communautaire. Des répondants et répondantes soulignent l'importance d'implanter des solutions qui se feront sentir à la base, sur le plan systémique. En même temps, ces répondants ont affirmé l'importance de répondre aux besoins immédiats des personnes concernées. Selon Jean-Michel, il faut ainsi s'attaquer aux discriminations dans le domaine de l'emploi, de l'embauche et du maintien en emploi. L'intervention doit également se faire avec les personnes les plus concernées: il faut les outiller afin qu'elles passent à l'action. Jacques et Claire, deux intervenants communautaires, suggèrent en outre une approche d'accompagnement qui prenne en compte les identités multiples afin de mieux servir les personnes LGBTQ immigrantes et racisées :

L'accompagnement des nouveaux individus se fait au rythme de la personne. Nous avons constaté que la flexibilité désamorce le stress et facilite le processus d'intégration chez la personne. (Jacques)

\section{Rendre accessibles des services spécialisés à la population vulnérable}

Une série de pistes sont proposées par des participants: l'accroissement des ressources spécialisées en hébergement, l'aide à la régularisation du statut d'immigration ou de réfugié, le développement des organismes spécialisés, la création d'un centre culturel (un espace multiservices) pour les personnes LGBTQ racisées, l'amélioration de la connaissance des enjeux touchant les personnes LGBTQ racisées, le fait de rendre visibles des modèles LGBTQ racisées, de rendre accessible le soutien psychosocial spécialisé.

Parlant des défis des personnes LGBTQ racisées, un intervenant mentionne l'importance de bien saisir les lacunes ${ }^{4}$ de la Loi sur l'immigration et la protection des réfugiés (LIPR), mais surtout de répondre aux besoins qu'éprouvent tous les jours les demandeurs d'asile. Spécifiquement pour les demandeurs d'asile LGBTQ, le manque d'expérience des professionnels représente un obstacle majeur à l'accès à un service spécialisé. Une intervenante confirme que la création d'une ressource spécialisée serait un atout pour cette population :

Il y a tellement de services spécialisés pour les femmes victimes de violence conjugale... Je crois qu'un service spécialisé pour les demandeurs d'asile LGBT, ce n'est pas trop demander, parce que chaque problématique essaie de trouver ses propres ressources, d'une certaine façon. (Claire)

\section{Discussion et conclusion}

Dans le présent article, nous avons dressé un portrait des diverses barrières intersectionnelles vécues par des personnes LGBTQ racisées, notamment à travers leurs trajets migratoires et leurs réalités quotidiennes au Québec, dans les domaines de l'emploi, de l'hébergement et de l'accès aux soins de santé et aux services sociaux. Nous avons décrit leur perception des réseaux communautaires, des relations intracommunautaires et des rapports structurels et interculturels, la façon dont se conçoit une identité complexe. Les témoignages recueillis nous ont également permis de prendre connaissance des pistes de solution privilégiées par les répondants et répondantes pour remédier aux déficiences constatées. L'analyse des résultats nous amène à faire plusieurs constats à propos de la diversité des parcours et des identités, de la faible représentation médiatique des personnes LGBTQ racisées, du rôle des organismes ainsi que de la présence de racisme systémique au Québec.

\footnotetext{
${ }^{4}$ Parmi les lacunes dénoncées, on retrouve l'entente sur les tiers pays sûrs qui empêche des individus arrivant des É.U. de demander refuge aux frontières terrestres canadiennes. Pour l'éventail des lacunes de la LIPR par rapport aux besoins des demandeurs et demanderesses d'asile LGBTQ, voir La Violette (2009) et Lee et Brotman (2011).
} 


\section{Des parcours divers et des identités multiples}

L'analyse des réponses des participants et participantes permet de poser divers constats en ce qui concerne leurs conceptions de l'identité. Ayant comme toutes les autres personnes LGBTQ à se forger une identité dans un contexte minoritaire où certains rapports de pouvoir les poussent à ne pas se reconnaître dans le discours hétéronormatif et cisnormatifs, et quelles que soient leurs caractéristiques, elles montrent un attachement aux différents pôles identitaires, entre autres sexuel, de genre, culturel, familial et religieux. La tendance dominante est que les répondants ne voient pas de contradictions dans cette identité multiple, ils y perçoivent plutôt un enrichissement. Or les expériences vécues ou perçues de discrimination, d'exclusion et de rejet, autant dans leur communauté d'origine que dans la société en général, les renvoient constamment à leur différence ou à leur altérité et influent sensiblement sur le processus complexe de leur construction identitaire. Le regard qu'offre l'approche intersectionnelle peut nous aider à mieux comprendre les réalités des personnes LGBTQ racisées en appuyant l'idée d'une construction identitaire multiple et holistique.

\section{Une faible représentation dans les médias}

Les personnes LGBTQ racisées sont très peu représentées dans les médias québécois, pour ne pas dire quasi absentes. Selon plusieurs, une telle absence contribue grandement à perpétuer, dans les représentations sociales, et cela d'une façon erronée, la perception d'une homogénéité culturelle. Cette quasi-absence médiatique est, selon ces mêmes participantes, préjudiciable aux jeunes racisés, dans la mesure où elle les prive de modèles auxquels ils pourraient fièrement s'identifier. En effet, "les personnes racisées sont souvent exclues du "nous" dans les représentations du groupe majoritaire ॥ (CDPDJ, 2011, p. 15). Elles sont plus susceptibles d'être tenues à l'écart de la vie citoyenne et, lorsqu'elles s'y insèrent, elles doivent surmonter des obstacles supplémentaires pour accéder aux mêmes chances que les personnes non racisées.

\section{Le rôle des organismes communautaires en matière d'intégration}

Le travail des associations LGBTQ actives auprès des immigrants en matière d'intégration est plutôt critiqué par plusieurs des interviewés, y compris ceux et celles étant affiliés aux organismes communautaires LGBTQ, à cause d'une déficience structurelle, de problèmes de financement, de la méconnaissance de ces associations par les personnes immigrantes et ou racisées et de l'absence de réseaux les liant aux autres communautés. Les répondants ont également souligné ce que les organismes communautaires œuvrant auprès des immigrants peuvent faire afin de s'attaquer à l'homophobie et à la transphobie au sein des communautés immigrantes et racisées. Plusieurs ont exprimé leur désir que des programmes et services spécialisés répondent aux expériences et au vécu particulier des personnes LGBTQ réfugiées.

\section{Une discrimination systémique soutenue}

Les personnes racisées, en particulier les personnes LGBTQ racisées, sont surreprésentées dans les strates les plus défavorisées de la population. Selon Statistique Canada, parmi les Québécois âgés de 15 ans et plus détenteurs d'un diplôme universitaire, le taux de chômage est de 3,7 \% chez les personnes n'appartenant pas à une « minorité visible ", contre 11,9\% chez les membres des " minorités visibles " (MICC, 2013). Quant à la discrimination systémique, elle englobe la discrimination directe et indirecte, mais va beaucoup plus loin. Elle repose sur l'interaction dynamique entre des décisions et des attitudes teintées de préjugés, ainsi que sur des modèles et des pratiques organisationnelles et institutionnelles qui ont des effets d'exclusion. Les répondants et répondantes ont associé certaines expériences vécues au racisme systémique, en particulier sur le marché du travail et au sein des communautés LGBTQ. Ces expériences les amènent à ne pas se considérer comme citoyens à part entière ainsi qu'à se réapproprier et à revendiquer une identité LGBTQ racisée. Loin d'être une société sans discrimination, nos résultats indiquent que les situations de discrimination vécues par les personnes LGBTQ s'inscrivent dans une logique qui, comme le dit Salée (2016), « nourrit tacitement une hiérarchie sociale racisante qui construit le porteur de différence ethnoculturelle en tant qu'Autre moins bon, moins valable, sinistre, menaçant, qu'il faut avoir à l'œil » (p. 270). 


\section{Conclusion}

Cet article ne peut prétendre résoudre la multitude de problèmes rencontrés par les personnes LGBTQ racisées en matière d'intégration citoyenne, professionnelle ou autre. II permet néanmoins de mieux les cerner et pourra provoquer, du moins nous l'espérons, une réflexion sérieuse à ce sujet. Des interventions plus directes, dont l'accès à l'éducation contre l'homophobie et la transphobie, à des formations interculturelles et intraculturelle, constituent les principaux piliers des solutions à envisager.

En mettant en lumière l'héritage colonial de la criminalisation de la sexualité et du genre non normative, nous récupérons un morceau de l'histoire oubliée de la violence sociale. Cette récupération de l'histoire reconfigure les connaissances sur les origines de l'homophobie et de la transphobie vécues actuellement. Ces histoires locales et mondiales situent les actes homophobes et transphobes au sein des héritages coloniaux, des nationalismes postcoloniaux et d'un ordre économique et politique mondial inégal. Les continuités historiques de ces héritages coloniaux et impériaux façonnent intimement la violence sociale contemporaine.

Les répondants et répondantes proposent plusieurs solutions afin de lutter contre les barrières: éducation interculturelle, plus grande participation sociale et visibilité accrue des personnes LGBTQ racisées qui constituent des modèles de réussite, intégration par le travail, etc. Des interventions plus directes, dont l'accès à l'éducation contre les discriminations intersectionnelles et les barrières multiples, forment les principaux piliers des solutions à envisager. Des activités de sensibilisation appuyées par le gouvernement, les institutions et la société québécoise, à la fois en tant qu'individus et que groupes communautaires, sont jugées comme des actions nécessaires pour éliminer les stéréotypes et promouvoir des relations interculturelles plus harmonieuses. À cet égard, les médias sont appelés à jouer un rôle plus objectif et responsable en matière de visibilité des personnes LGBTQ racisées, étant donné leur pouvoir de façonner les perceptions. Par ailleurs, la mise en œuvre de certains services doit être améliorée, notamment lorsqu'il s'agit de services spécialisés d'accompagnement et d'aide psychosociale. Cela dit, médias, gouvernements, organisations et individus sont incités à agir concrètement afin de conjuguer leurs efforts pour relever les défis des relations harmonieuses au sens large.

Si les limites qu'impose notre échantillon, restreint à 23 participants, rendent impossible toute généralisation, nos résultats revêtent une importance théorique et sociale indéniable. De plus, cette recherche exploratoire initiale nous incite à entrevoir plusieurs pistes de recherche pour le futur. II serait important d'envisager une recherche de plus grande envergure, en incluant des personnes habitant dans d'autres régions canadienne, afin de dresser un tableau comparatif et global de la situation des personnes LGBTQ racisées au Canada. D’autres pistes de recherche peuvent aussi être envisagées dans un cadre institutionnel spécifique, par exemple, les expériences des jeunes LGBTQ racisés dans les écoles et cégeps. Aussi, approfondir nos connaissances sur les pratiques en intervention sociale et l'accès aux soins de santé et des services sociaux de cette population aiderait certainement les personnes intervenantes à développer des outils sur le plan micro-systémique afin de mieux répondre aux besoins d'encadrement social et thérapeutique des personnes LGBTQ racisées.

\section{Références bibliographiques}

Alexander, M. (2005). Pedagogies of Crossing: Meditations on Feminism, Sexual Politics, Memory and the Sacred. Londres: Duke University Press.

Alexander, M. et Mohanty, C. (1997). Feminist Genealogies, Colonial Legacies, Democratic Futures. New York : Routledge.

Bilge, S. (2009). Théorisations féministes de l'intersectionnalité. Diogène, 1(225), 70-88.

Bilge, S. et Denis, A. (2010). Introduction: Women, intersectionality and diasporas. Journal of Intercultural Studies, $31(1), 1-8$.

Carter, S. (2008). The Importance of being monogamous: Marriage and nation building in Western Canada to 1915. Edmonton : University of Alberta Press. 
Chbat, M. (2011). Articulations et négociations des identifications ethno-sexuelles des gais et des lesbiennes d'origine libanaise à Montréal (Mémoire de maîtrise en sociologie non publié). Université de Montréal.

Cohen, S. (2008). The gay liberation youth movement in New York: An army of lovers cannot fail. New York : Routledge.

Commission des droits de la personne et des droits de la jeunesse du Québec (CDPDJ). (2011). Profilage racial et discrimination systémique des jeunes racisés (Rapport de recherche). Montréal : Direction de la recherche, de l'éducation-coopération et des communications.

Crenshaw, K. (1996). Mapping the Margins : Intersectionality, Identity Politics, and Violence Against Women of Color. Dans K. Crenshaw, N. Gotanda, G. Peller et K. Thomas (dir.), Critical Race Theory : They Key Writings That Formed the Movement (p. 357-383). New York : The New Press.

Demczuk, I. et Remiggi, F. (1998). Sortir de l’ombre : histoires des communautés lesbienne et gaie de Montréal. Montréal : VLB Éditeur.

Duggan, L. (2003). The Twilight of Equality? Neoliberalism, Cultural Politics, and the Attack on Democracy. Boston : Beacon Press.

Ekine, S. (2013). Contesting narratives of queer Africa. Dans S. Ekine et H. Abbas (dir), Queer African Reader. Dakar : Pambazuka Press.

Enriquez, M. (2013). La contestation des politiques de changement d'identité de genre par les militantes et militants trans québécois. Lien social et Politiques, 69, 181-196.

Espin, O. (1997). Crossing Borders and Boundaries : The Life Narratives of Immigrant Lesbians. Dans B. Greene (dir.), Ethnic and cultural diversity among lesbians and gay men (191-215). Californie : Sage Publications.

Gagné, F. et Chamberland, L. (2008). Parcours migratoires et identités gaies et lesbiennes. Dans S. Brotman et J.J. Lévy (dir.), Intersections : cultures, sexualités et genres (p. 159-192). Québec: Presses de l'Université du Québec.

Guillaumin, C. (1972). L'idéologie raciste : genèse et langage actuel. La Haye : Mouton.

Gupta, A. (2008). This alien legacy: The origins of "sodomy" laws in British Colonialism.

Human Rights Watch (2008). The Alien Legacy. The Origins of "Sodomy" Laws in British Colonialism. New York: New York. En ligne à : http://www.hrw.org/reports/2008/12/17/alien-legacy-0

Haritaworn, J. (2012). Colorful bodies in the multikulti metropolis: Vitality, victimology and transgressive citizenship in Berlin. Dans T. Cotton (dir.), Transgender migrations : The bodies, borders, and politics of transition (p. 1131). New York, NY : Routledge.

Kapac, J. (1998). Culture/Community/Race : Chinese Gay Men and the Politics of Identity, Anthropologica, 40(2), 169-182.

Kinsman, G. (1998). Constructing sexual problems: These things may lead to the tragedy of our species. Dans W. Antony et L. Samuelson (dir.), Power and resistance: Critical thinking about Canadian social issues ( $2^{\mathrm{e}}$ éd., p. 256-282). Halifax : Fernwood Publishing.

La Violette, N. (2009). Independent human rights documentation and sexual minorities: An ongoing challenge for the Canadian refugee determination process. International Journal of Human Rights, 13, 437.

Lee, W. J. E. (2015). The social organization of queer / trans migrations: The everyday experiences of queer and trans migrants with precarious status. Montréal : Université McGill.

Lee, E. et Brotman, S. (2011). Identity, refugeeness, belonging: Experiences of sexual minority refugees in Canada. Canadian Review of Sociology, 48(3), 241-274.

Lowe L. (2006). The intimacies of four continents. Dans L. Stoler (dir.), Haunted by empire: Geographies of intimacy in North American intimacy (p. 191-212). Durham et London : Duke University Press.

Alterstice - Revue Internationale de la Recherche Interculturelle, vol. $6, n^{\circ} 2$ 
Meyer-Cook, F. et Labelle, D. (2004). Namaji: Two-Spirit Organizing in Montreal, Canada. Journal of Gay and Lesbian Social Services, 16(1), 29-51.

Miles, R. (1993). Racism after "Race relations". New York Routledge.

Ministère de l'immigration et des communautés culturelles (MICC) (2013). Présence en 2013 des immigrants admis au Québec de 2002 à 2011. Québec : Gouvernement du Québec. En ligne à http://www.midi.gouv.qc.ca/publications/fr/recherchesstatistiques/PUB_Presence2013_admisQc_02_11.pdf

O’Brien, C.-A., S. Abualsameed, H., Heung, F., Ridgley, A. et Scanlon, K. (2005). International Migration : Newcomer and Refugee Youth. Dans Youth Migration Project. Toronto : AIDS Committee of Toronto.

Ossome, L. (2013). Postcolonial discourses of queer activism and class in Africa. Dans S. Ekine et H. Abbas. (dir.), Queer African reader (p. 32-47). Dakar : Pambazuka Press.

Ricard, N. (2011). Le prix à payer pour devenir sujet de droit : la sélection des réfugiées allosexuels au Canada. Alterstice, 1(2), 79-96.

Pitt-Rivers, J. (1966). Honour and social status. Dans J. Peristiany (dir.), Honour and Shame (p. 19-77). Chicago : University of Chicago Press.

Puar, J. (2007). Terrorist assemblages : Homonationalism in queer times. Durham : Duke University Press.

Roy, O. (2013). Homme immigrant cherche homme : (re)formations de subjectivités ethnosexuelles en contexte post-migratoire au Québec (Thèse de doctorat non publiée). Université de Montréal.

Salée, D. (2016). Vivre-ensemble et dynamiques de pouvoir : éléments pour comprendre l'anxiété antipluraliste actuelle des Québécois. Dans A-G. Gagnon et J.-C. St-Louis (dir.), Les conditions du dialogue au Québec : Laïcité, réciprocité, pluralisme (p. 253-282). Montréal : Québec Amérique.

White, M. (2010). Intimate archives, migrant negotiations: Affective governance and the recognition of "same-sex" family class migration in Canada (Thèse de doctorat non publiée). Université York. 